\title{
Germinación de semillas de espinaca (Spinacia oleoracea L.) utilizando tres sustratos en la región Amazonas
}

\section{Germination of spinach seeds (Spinacia oleoracea L.) using three substrates in the Amazon region}

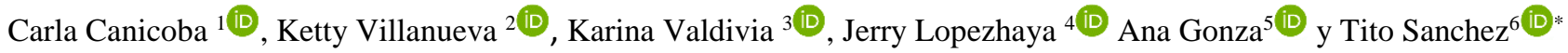

\begin{abstract}
RESUMEN
El presente trabajo tuvo por objetivo evaluar la germinación de semillas de espinaca (Spinacia oleoracea L.) utilizando tres sustratos en la región Amazonas. Se instaló bajo un diseño completo al azar con 3 tratamientos (T1: tierra agrícola, T2: turba y T3: arena), 3 repeticiones y 100 semillas por unidad experimental. Se construyó tres germinadores de madera con dimensiones $60 \mathrm{~cm} \mathrm{x} 60 \mathrm{~cm}$ ancho y largo respectivamente. Se hicieron pequeños surcos en los cuales se colocaron a chorro continuo 100 semillas por repetición, luego se cubrió con plástico para mantener una temperatura y humedad relativa constante. Se encontró que las semillas de espinaca mostraron mayor porcentaje de germinación en sustrato tierra agrícola (63.33\%), seguido por arena (41.67\%), así mismo turba presentó el más bajo porcentaje (30\%). El tiempo promedio de germinación de espinaca en sustrato tierra agrícola, fue en 5.1 días y velocidad de germinación (33.17\%). Semillas en turba demoraron en germinar (6 días), siendo éste además el tratamiento que obtuvo menores valores en todas las variables evaluadas. El sustrato adecuado para germinar espinaca es tierra agrícola, así mismo, turba no es un sustrato recomendable para almacigados de semillas de espinaca.
\end{abstract}

Palabras clave: arena, porcentaje de germinación, tierra agrícola, turba, velocidad de germinación.

\begin{abstract}
The present work aimed to evaluate the germination of spinach (Spinacia oleoracea L.) seeds using three substrates in the Amazon region. It was installed under a complete random design with 3 treatments (T1: agricultural land, T2: peat and T3: sand), 3 repetitions and 100 seeds per experimental unit. Three wooden germinators were built with dimensions $60 \mathrm{~cm}$ x $60 \mathrm{~cm}$ width and length respectively. Small furrows were made in which 100 seeds per repetition were placed in a continuous stream, then covered with plastic to maintain a constant temperature and relative humidity. It was found that spinach seeds showed the highest percentage of germination in agricultural soil substrate $(63.33 \%)$, followed by sand $(41.67 \%)$, likewise peat presented the lowest percentage (30\%). The average germination time of spinach in agricultural soil substrate was 5.1 days and germination speed (33.17\%). Seeds in peat took a long time to germinate (6 days), this being also the treatment that obtained the lowest values in all the variables evaluated. The adequate substrate to germinate spinach is agricultural land, likewise, peat is not a recommended substrate for spinach seed storage.
\end{abstract}

Keywords: sand, germination percentage, agricultural land, peat, germination rate.

DOI: https://doi.org/10.37787/pakamuros-unj.v8i3.138

Recibido: 09/10/2020. Aceptado: 24/10/2020

* Autor para correspondencia

1. Facultad de Ingeniería y Ciencias Agrarias, Universidad Nacional Toribio Rodríguez de Mendoza de Amazonas, Calle Higos Urco N 342 - 350 - 356, Amazonas, Perú. E-mail: canicobarubio03@ gmail.com; 2. E-mail: k71130678v@gmail.com 3. E-mail:kmedaly.1997@gmail.com 4. E-mail:jerry.blm1998@ gmail.com 5. Email:anitagonzasaavedra@gmail.com 6. E-mail:titosanchezsantillan@gmail.com 


\section{INTRODUCCIÓN}

La espinaca (S. oleracea L.) se distribuye generalmente en Europa, Asia y parte de América, siendo el mayor productor a nivel mundial China, aproximadamnte 90\%, seguido por Japón y Estados Unidos (Agrocadenas, 2006).

En Perú, la espinaca se cultiva en Cañete, Chancay - Huaral, Lima y Tarma; cultivada en pequeñas extensiones en épocas de otoño e invierno. El Ministerio de Agricultura reporta que el año 1999 fueron producidas 9273 toneladas en 677 hectáreas, con rendimiento promedio de 13698 kilogramos por hectárea (Sigueñas, 2002). Evidenteente, la producción de espinaca está en crecimiento, tanto en superficie como en productividad con un rendimiento promedio anual de 14.9 t/ha (Webb y Fernández, 2000). El cultivo de espinaca, en relación con otros alimentos, es consumido eventualmente como ensalada fresca y permite generar ingresos dentro de la cadena productiva de las familias de escasos recursos, así mismo es muy importante desde el punto de vista nutritivo y de balance dietético en la alimentación de muchas familias (Hoyos et al., 2009).

Pese a su importancia, son escasas las investigaciones en este cultivo, debido a que se siembra en rotación con cultivos de más largo periodo y se cultiva en pequeñas áreas (Siura, Montes y Dávila, 2016).

En la región Amazonas y parte del Perú, la espinaca en su mayoría hacen siembra directa, no obstante, podría ser una desventaja, ya que se utiliza más semillas, genera mayor deshuniformidad en la emergencia y si no se utilizan semillas con buen valor cultural se podría perder el cultivo. En efecto, Surco \& Camacho (2019), recomiendan que se debería realizar un almacidgado previo con una combinación de sustratos (50\% suelo de lugar, $10 \%$ arena, $20 \%$ turba y $20 \%$ humus de lombriz), y luego trasplantarlas cuando tegan dos hojas verdaderas. También, un sustrato como fibra de coco resultaría favorable para realizar germinaciones (Jimenez et al., 2010).

En investigaciones en hortalizas, Miranda (2014), reportó que, en sustratos tierra negra y estiércol obtuvo mayor cantidad de plantines seguido de la mezcla tierra agrícola y tierra negra.

En este contexto, la presente investigación planteó evaluar la germinacion de espinaca utilizando tres sustratos, con miras a una transferencia hacia las familias rurales para instlación de biohuertos sostenibles. 


\section{MATERIALES Y MÉTODOS}

\section{Área de estudio}

La presente investigación se realizó en el distrito San Isidro del Maino, provincia Chachapoyas, región Amazonas con coordenadas $6^{\circ} 20^{\prime} 13^{\prime \prime} \mathrm{S} 77^{\circ} 52^{\prime} 59^{\prime \prime} \mathrm{O}$ y altitud de 2325 m.s.n.m.

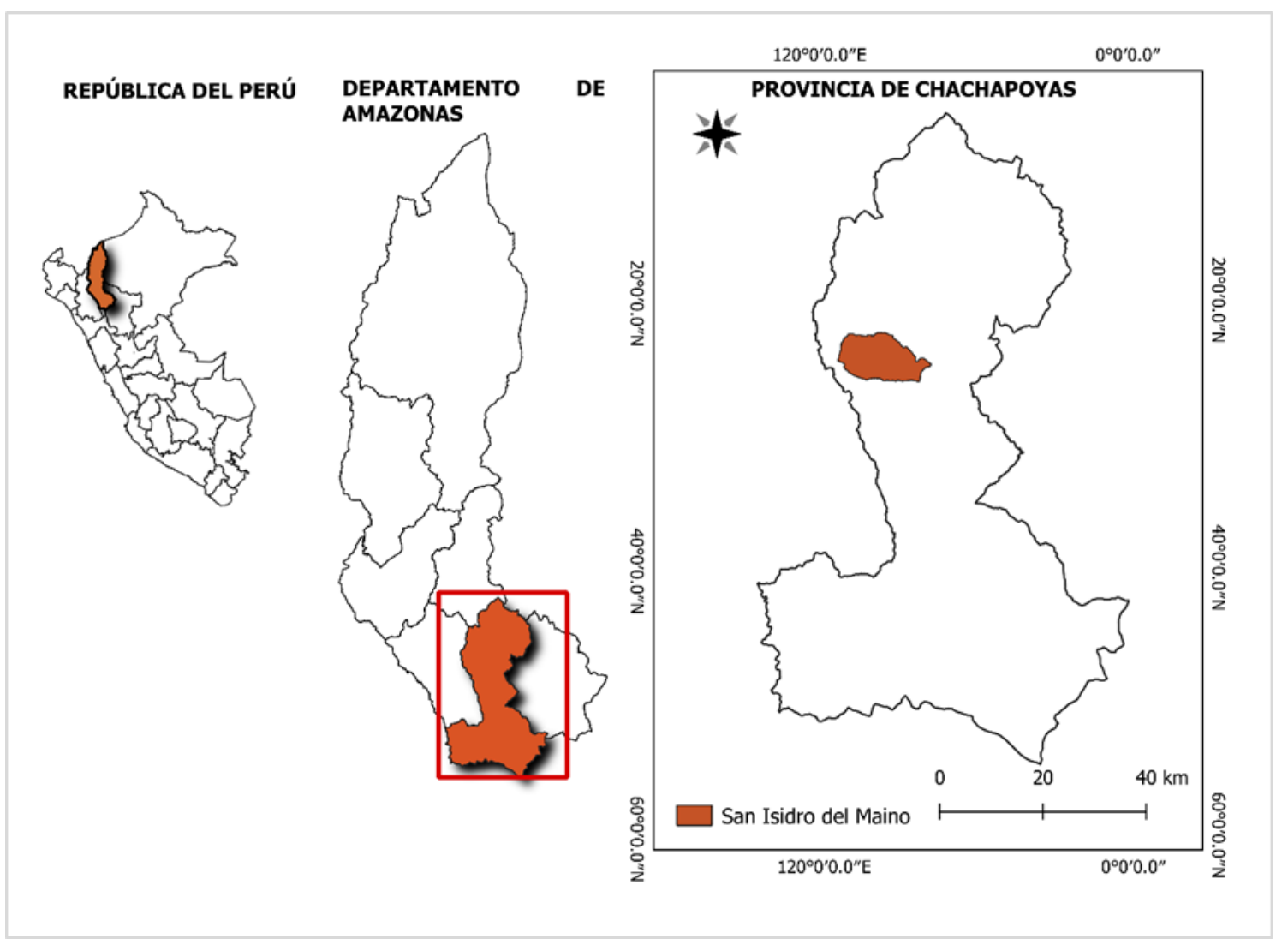

Figura 1. Mapa ubicación del área de investigación, distrito San Isidro del Maino, región Amazonas

El material utilizado fueron tres sustratos colectados del distrito San Isidro del Maino (Tabla 1), los mismos que fueron desinfectados con agua hervida $\left(100^{\circ} \mathrm{C}\right)$, en una carretilla por 10 minutos, repitiendo la operación tres veces. Finalmente, éstos se dejaron en plásticos para eliminar el exceso de agua y estar en capacidad de campo. 
Tabla 1. Descripción de tratamientos en la germinación de S. oleracea L.

\begin{tabular}{cc}
\hline Tratamientos & Muestra de sustrato \\
\hline $\mathrm{T} 1$ & Tierra agrícola \\
$\mathrm{T} 2$ & Turba \\
$\mathrm{T} 3$ & Arena \\
\hline
\end{tabular}

\section{Instalación de la investigación}

Se construyó tres cajones de madera con tamaños de 60 × 60 × $20 \mathrm{~cm}$, en las mismas se colocaron tres sustratos previamente desinfectados (tierra, turba y arena). En la base de los cajones se colocó un plástico para no tener contacto con la superficie de suelo, así mismo se realizaron unos agujeros para drenar el exceso de humedad. Las semillas se contabilizaron separándolas de 100 unidades, para cada repetición. Las semillas fueron colocadas en hileras, tapándolas con una capa fina del mismo sustrato en estudio. Se aplicó un riego nebulizado con una mochila fumigadora (capacidad 20 L), para humedecer el sustrato. Finalmente se cubrió con un plástico para mantener una temperatura y humedad constante, por 72 horas.

\section{Variables evaluadas}

Porcentaje de germinación: se contabilizaron semillas germinadas, luego se trasformaron en un formato Excel dividiendo el número germinado entre el total de semillas colocadas multiplicado por 100.

Para el coeficiente de velocidad de germinación se utilizó la ecuación (1).

$$
\mathrm{CV}=\frac{\sum \mathrm{n}_{\mathrm{i}}}{\sum\left(\mathrm{n}_{\mathrm{i}} \mathrm{t}_{\mathrm{i}}\right)} \times 100
$$

Para el tiempo promedio de germinación se utilizó la ecuación (2).

$$
\mathrm{T}=\frac{1}{\mathrm{CV}}
$$

Para el índice de germinación se utilizó la ecuación (3).

$$
\text { IG }=\frac{\sum\left(n_{i} t_{i}\right)}{N}
$$

Para la velocidad de germinación se utilizó la ecuación (4).

$$
M=\sum\left(\frac{n_{i}}{t}\right)
$$

Dónde:

$\mathrm{n}_{\mathrm{i}}=$ número de semillas germinadas al día $\mathrm{i}$, 
$\mathrm{t}_{\mathrm{i}}=$ número de días después de la siembra,

t=tiempo de germinación desde la siembra hasta la germinación de la última semilla,

$\mathrm{N}=$ Número total de semillas sembradas,

$\mathrm{CV}=$ Coeficiente de velocidad de germinación

$\mathrm{T}=$ Tiempo promedio de germinación,

IG=Índice de germinación,

$\mathrm{M}=$ Velocidad de germinación

\section{Diseño experimental y análisis de datos}

Se instaló bajo un diseño completo al azar con 3 tratamientos, 3 repeticiones, y 100 semillas por repetición. Para el porcentaje de germinación se realizó el cumplimiento de supuestos de normalidad (Shapiro Wilk) y homogeneidad de varianzas (Test de Levene). Cumplidos éstos se realizó el análisis de varianza y comparación múltiple de medias con el test post hoc de Tukey al 5\% de nivel de significancia. Se utilizó el software estadístico InfoStat versión 2017.

La Tabla 2, muestra el análisis de varianza para la germinación de semillas de espinaca influenciados por los sustratos; reportando que existe diferencias significativas entre los tratamientos estudiados (p-valor $<0.05)$.

Tabla 2. Análisis de varianza para la germinación de espinaca influenciado por sustratos

\begin{tabular}{llllll}
\hline F.V & SC & gl & CM & F & p-valor \\
\hline Tratamiento & 1716.67 & 2 & 858.33 & 10.88 & $0.0101^{*}$ \\
Error & 473.33 & 6 & 78.89 & & \\
Total & 2190.00 & 8 & & & \\
\hline
\end{tabular}

*= diferencias significativas; SC: suma de cuadrados; gl: grados de libertad; CM: cuadrados medios; gl: grado de libertad; F: Fisher

\section{RESULTADOS}

La Tabla 3, muestra el análisis de varianza para la germinación de semillas de espinaca influenciados por los sustratos; reportando que existe diferencias significativas entre los tratamientos estudiados (p-valor $<0.05)$. 
Tabla 3. Análisis de varianza para la germinación de espinaca influenciado por sustratos

\begin{tabular}{llllll}
\hline F.V & SC & gl & CM & F & p-valor \\
\hline Tratamiento & 1716.67 & 2 & 858.33 & 10.88 & $0.0101^{*}$ \\
Error & 473.33 & 6 & 78.89 & & \\
Total & 2190.00 & 8 & & & \\
* = diferencias significativas; SC: suma de cuadrados; gl: grados de libertad; CM: cuadrados \\
medios; gl: grado de libertad; F: Fisher
\end{tabular}

En la Figura 2, se presenta la comparación múltiple de medias para la germinación de espinaca con el test de Tukey $(\mathrm{a}=0.05)$, dónde el T1 (tierra agrícola) mostró mayor porcentaje de germinación con diferencias estadísticas significativas, seguido por el T3 (arena), el tratamiento más bajo resultó el T2 (turba).

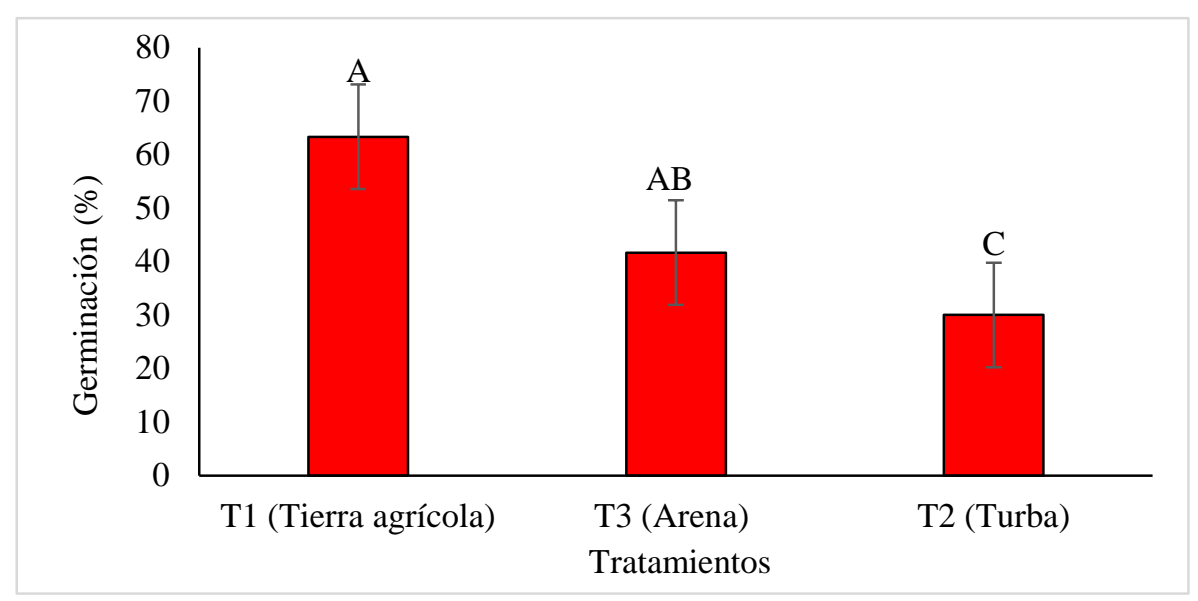

Figura 2. Test post hoc de Tukey $(\mathrm{a}=0.05)$ para la geminación total de semillas.

La Figura 3, muestra la curva de germinación; en el día 5 después de la siembra, el T1 presentó mayor número de semillas germinadas, no obstante, para el día 6 el T3 resultó ser superior, pero en menor cantidad que el día 5. En el séptimo día todos los tratamientos mostraron germinación homogénea, 
terminando el proceso en el día 8. La curva de germinación para el sustrato turba, se mantuvo constante y por debajo de los dos sustratos (Figura 3).

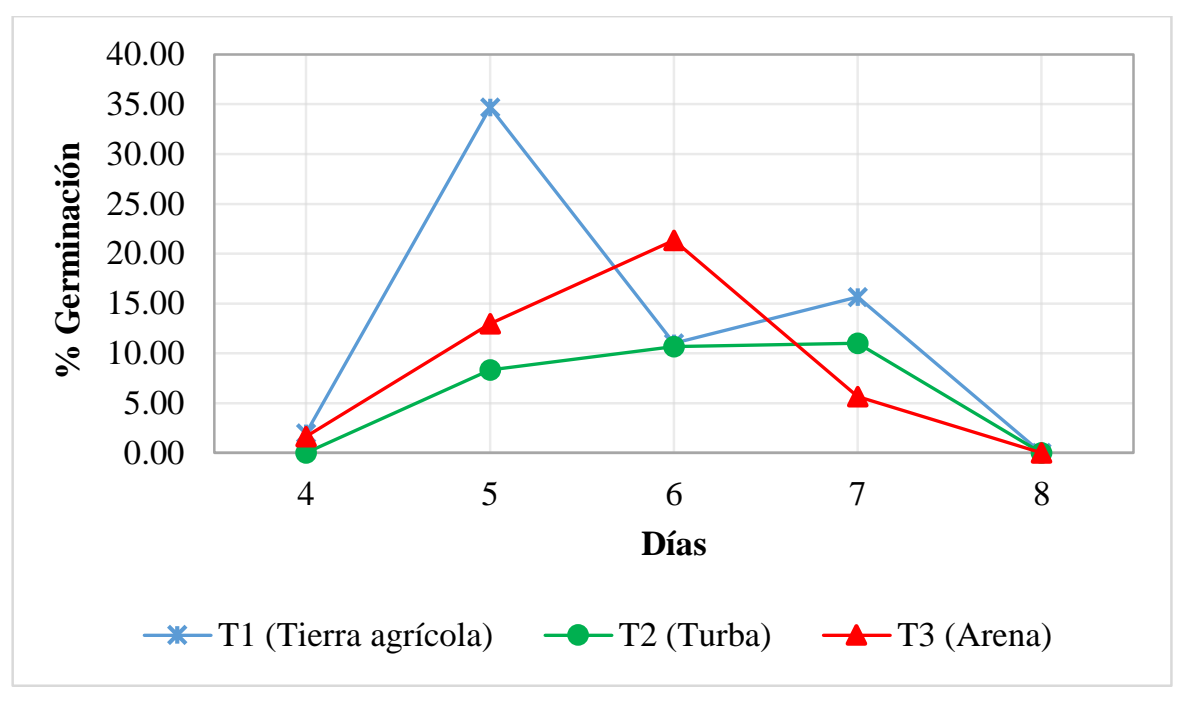

Figura 3. Curvas de germinación de semillas de S. oleracea L.

La Tabla 4, nos muestra que, el sustrato tierra agrícola mostró mayor índice y velocidad de geminación respecto a los demás; así mismo, el tiempo promedio de germinación fue en 5.8 días. El sustrato turba mostró valores inferiores en la velocidad e índice de germinación, pero fue mayor en el tiempo de germinación (6.3 días).

Tabla 4. Parámetros de germinación calculados

\begin{tabular}{lllll}
\hline \multicolumn{5}{c}{ Variables } \\
\hline Tratamiento & CV (\%) & TG & IG (\%) & VG (\%) \\
\hline T1 (tierra agrícola) & 17.2 & 5.8 & 63.3 & 33.7 \\
T2 (turba) & 16.3 & 6.1 & 19.3 & 9.7 \\
T3 (arena) & 16 & 6.3 & 41.7 & 20.6 \\
\hline
\end{tabular}

CV: coeficiente de velocidad de germinación; TG: tiempo de germinación; IG: índice de germinación; VG: velocidad de germinación 


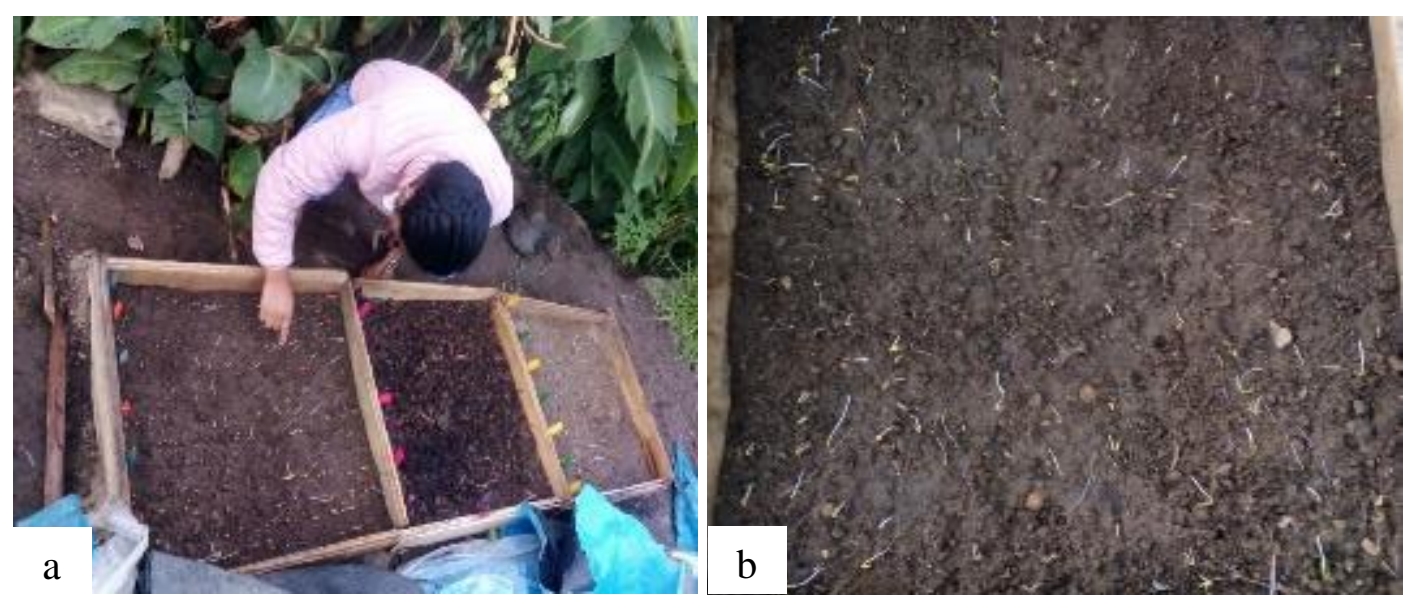

Figura 4. Evaluación a los 6 días después de siembra: a) Conteo de plantines emergidos de espinaca;

b) germinación en sustrato tierra agrícola

\section{DISCUSIÓN}

El porcentaje promedio de germinación en espinaca, fue mejor en el sustrato tierra agrícola, seguido por arena, resultando ser el peor tratamiento turba (Figura 2). En efecto un sustrato moderadamente proporcional en sus estructuras favorecería positivamente la germinación. Sin embargo, Handreck y Black (2002), reportan que la porosidad total de un sustrato afecta la capacidad de intercambio gaseoso del medio, disminuyendo el contenido de oxígeno que las semillas requieren para germinar. Así mismo, los sustratos con alto contenido de abono orgánico, tienen menor espacios porosos, ende baja capacidad de circulación de aire.

Hartmann y Kester (1971), menciona que la demora de la emergencia y el bajo \% de germinación en ocasiones pueden estar afectados por factores internos de las semillas (viabilidad del embrión, la cantidad y calidad del tejido de reserva y los diferentes tipos de dormancia), así mismo, factores externos como (temperatura, humedad, aireación, etc.), probablemente esto pudo ocurrir en la espinaca ya que el porcentaje de germinación no superó el $80 \%$.

Méndez, Moreno y Moya (2009), en investigaciones sobre germinación de semillas pequeñas, mencionan que en arena obtuvieron mayor germinación en comparación con mezcla de sustrato (arena + suelo) y suelo puro, reportando que el sustrato suelo presentó las peores condiciones para la germinación, afectado en parte también a la combinación (arena + suelo); esto es reforzado por Sigueñas (2020), dónde recomiendan sustrato arena para hacer un almacigado o prueba de viabilidad en semillas pequeñas. No obstante, todo lo contrario, se obtuvo en la presente investigación, ya que las semillas de espinaca germinaron mejor en tierra agrícola, superando ampliamente a sustrato arena que solo logró un 41.7\%. 
Miranda (2014), evaluó formulaciones de sustratos en base a turba, para almácigos de hortalizas en ambiente controlado, concluyendo que en tierra negra y estiércol obtuvieron mayor cantidad de plantines, seguido por la mezcla tierra agrícola + tierra negra, reforzando a lo encontrado, dónde tierra agrícola resultó mejor.

Por su parte, Ibáñez (2006), menciona que suelos de textura fina presentan baja tasas de germinación, ya que retienen mayor cantidad de agua, por ende, generan microclimas de alta humedad y causan la pudrición de las semillas.

Respecto a la curva de germinación, las semillas de espinaca tardaron en germinar 7 días en los tres sustratos evaluados (Figura 3). En el día 5 las semillas en tierra agrícola mostraron mayor número de semillas germinadas. Sin embargo, en el día 6 éste bajó drásticamente, y la germinación en arena mostró el mayor germinado. Semillas en turba mostraron una germinación constante y en menor porcentaje (Figura 3).

Los resultados obtenidos son similares a los reportados por Roldán \& Soto (2005), en semillas de lechuga y brócoli donde las semillas tardaron en germinar y emerger entre 4 y 6 días. Mencionando a su vez que, es común que las semillas pequeñas, por naturaleza tienen poca reserva, y en ocasiones tardan en germinar especialmente en sustratos de mayor densidad. Esta afirmación y comparación es aplicable sólo en hortalizas, ya que en su mayoría éstos tienen una germinación rápida, comparados con semillas de otras especies.

La velocidad de germinación (33.7\% de espinaca y menor tiempo (5 días) estuvo influenciado por el sustrato tierra agrícola. El sustrato turba mostró el menor valor en las variables evaluadas, resultando como un sustrato no adecuado para germinar esta hortaliza. Esto puede ajustarse a lo que menciona Ibáñez (2006), dónde los sustratos con textura fina, retienen más humedad causando pudrición de las semillas; característica particular de los sustratos turbosos.

\section{CONCLUSIONES}

Las semillas de espinaca presentaron mayor porcentaje de germinación en tierra agrícola, así mismo el sustrato turba fue el tratamiento con menor número de semillas germinadas. La germinación inició a partir quinto día, obteniéndose que tierra agrícola logró la máxima germinación en el día 6. El sustrato turba presentó una germinación constante en todos los días, además presentó valores por debajo de los sustratos tierra agrícola y arena. El mejor sustrato para almacigar la espinaca es tierra agrícola; no se recomienda utilizar sustrato turba, ya que reportó bajo porcentaje de germinación. 


\section{REFERENCIAS BIBLIOGRÁFICAS}

Agrocadenas, O. (2006). Agroindustria y competitividad. Bogotá: Diseño y Coordinación Editorial.

Handreck, K. A., Black, N. D., \& Black, N. (2002). Growing media for ornamental plants and turf. UNSW press.

Hartmann, H., \& Kester, E. (1971). Propagación de plantas. Mexico D.F: Editorial Continental.

Hoyos, V., Rodríguez, M., Cárdenas-Hernández, J. F., \& Balaguera-López, H. E. (2009). Análisis del crecimiento de espinaca (Spinacia oleracea L.) bajo el efecto de diferentes fuentes y dosis de nitrógeno. Revista Colombiana de Ciencias Hortícolas, 3(2), 175-187.

Ibáñez, J. J. (2006). EL agua en el Suelo 4: Textura del Suelo y Propiedades Hídricas. Un Universo invisible bajo nuestros pies. Los suelos y la vida [en línea]. Disponible en: https://www.madrimasd.org/blogs/universo/2006/07/05/33887

Jimenez, J., Fuentes, L., Niño, N., Espinoza, L., Arias , L., Rodriguez , M., \& Garzón, C. (2010). El cultivo de la espinaca(Spinacia oleraceaL.)y su manejo fitosanitarioen Colombia. Bogota: Fundación de la Universidad de Bogota . Recuperado el 22 de 07 de 2020, de http://avalon.utadeo.edu.co/servicios/ebooks/espinaca/files/assets/basic-html/page3.html

Méndez Natera, J., Moreno, M., \& Moya, J. (2009). Efecto de diferentes combinaciones de sustratos (arena, suelo y/o bagazo de caña de azúcar) sobre la germinación de semillas y altura de plantas de guayaba (Psidium guajava L.). UDO Agrícola, 9(1), 121-125. Obtenido de file:///C:/Users/Usuario/Downloads/Dialnet-

EfectoDeDiferentesCombinacionesDeSustratosArenaSue-3293836\%20(1).pdf

Miranda Balboa , G. (2014). Evaluación de sustrato en base a turba en ambiente protegido, para producción de almacigo horticola en invernadero, en el municipio de El Alto. Universidad Nacional de San Andrés , Facultad de Agrónomia . La paz : Repositorio Institucional . Obtenido de http://hdl.handle.net/123456789/4289

Roldán, G. Q., \& Soto, C. M. (2005). Evaluación de sustratos para almácigos de hortalizas. Agronomía mesoamericana, 16(2), 171-183. 
Saavedra, S. S. (2002). Cultivo de Espinaca (Primera ed., Vol. 1). Lima: INIA. Obtenido de http://repositorio.inia.gob.pe/bitstream/inia/920/1/Folleto\%20-

\%20El\%20Cultivo\%20de\%20Espinaca\%20\%20R.I.\%202002.pdf

Sigueñas Saavedra, S. (2002). El cultivo de espinaca. Disponible en: http://repositorio.inia.gob.pe/bitstream/inia/920/1/Folleto\%20-

$\% 20$ El\%20Cultivo\%20de\%20Espinaca\%20\%20R.I.\%202002.pdf

Siura, S., Montes, I., \& Dávila, S. (2016, March). Efecto del biol y la rotación con abono verde (Crotalaria juncea) en la producción de espinaca (Spinacea oleracea) bajo cultivo orgánico. In Anales científicos (Vol. 70, No. 1, pp. 1-8).

Surco, H. V. Q., \& Camacho, E. C. (2019). Efecto de AOLA sobre el rendimiento a diferentes densidades de siembra del cultivo espinaca (Spinacea oleracea L.) en ambiente atemperado en el Centro Experimental Cota Cota. Apthapi, 5(1), 1415-1429. ISSN: 2519-9382

Webb, R., \& Fernández-Baca, G. (2000). Perú en números 2000. Lima: Cuánto SA. 